Research article

\title{
Correlation between CpG methylation profiles and hormone receptor status in breast cancers
}

\author{
Weiwei Feng1, Lanlan Shen², Sijin Wen³, Daniel G Rosen ${ }^{4}$, Jaroslav Jelinek², Xin Hu¹, \\ Shaoyi Huan1, Miao Huang1, Jinsong Liu${ }^{4}$, Aysegul A Sahin ${ }^{4}$, Kelly K Hunt ${ }^{5}$, Robert C Bast Jr1, \\ Yu Shen ${ }^{3}$, Jean-Pierre J Issa ${ }^{2}$ and Yinhua $\mathrm{Yu}^{1}$
}

\begin{abstract}
1Department of Experimental Therapeutics, The University of Texas MD Anderson Cancer Center, 1515 Holcombe Blvd, Houston, TX 77030, USA 2Department of Leukemia, The University of Texas MD Anderson Cancer Center, 1515 Holcombe Blvd, Houston, TX 77030, USA ${ }^{3}$ Department of Biostatistics, The University of Texas MD Anderson Cancer Center, 1515 Holcombe Blvd, Houston, TX 77030, USA ${ }^{4}$ Department of Pathology, The University of Texas MD Anderson Cancer Center, 1515 Holcombe Blvd, Houston, TX 77030, USA ${ }^{5}$ Department of Surgical Oncology, The University of Texas MD Anderson Cancer Center, 1515 Holcombe Blvd, Houston, TX 77030, USA
\end{abstract}

Corresponding author: Yinhua Yu, yyu@mdanderson.org

Received: 26 Apr 2007 Revisions requested: 1 Jun 2007 Revisions received: 13 Jul 2007 Accepted: 31 Aug 2007 Published: 31 Aug 2007

Breast Cancer Research 2007, 9:R57 (doi:10.1186/bcr1762)

This article is online at: http://breast-cancer-research.com/content/9/4/R57

(C) 2007 Feng et al.; licensee BioMed Central Ltd

This is an open access article distributed under the terms of the Creative Commons Attribution License (http://creativecommons.org/licenses/by/2.0), which permits unrestricted use, distribution, and reproduction in any medium, provided the original work is properly cited.

\begin{abstract}
Introduction Aberrant DNA methylation has been found frequently in human breast cancers, associated with the loss of expression of a number of regulatory genes for growth and correlated to clinical outcomes. The present study was undertaken to determine whether methylation of a set of growthsuppressor genes would correlate to the expression of estrogen receptors (ERs) and progesterone receptors (PRs).
\end{abstract}

Methods We used a pyrosequencing methylation analysis to study the methylation of 12 known growth-suppressor genes in 90 pairs of malignant/normal breast tissues. We also examined the expression of ERs and PRs in those specimens by immunohistochemistry. Mutations of $p 53$ in tumor cells were detected by direct sequencing.

Results Twelve tumor-suppressor genes: ARHI, RASSF1A, HIN-1, RAR 2 2, hMLH1, 14-3-3 $\sigma, R I Z 1, p 16$, E-cadherin, RIL, $C D H 13$, and $N K D 2$ were selected for this methylation study. Five of them (RIL, HIN-1, RASSF1A, CDH13, and RAR 32 ) were frequently methylated in breast cancers $(57 \%, 49 \%, 58 \%$, $44 \%$, and $17 \%$, respectively) but not the normal breast $(0-4 \%)$. Two panels of methylation profiles were defined. The methylation of the HIN-1/RASSFIA panel strongly correlated to the expression of ERs, PRs, and hormone receptors (HRs; which were defined as 'positive' if ERs and/or PRs were positive; $p<0.001$ ). Conversely, the methylation of the RILI $\mathrm{CDH} 13$ panel strongly correlated to negative ER, PR, and HR expression ( $p=0.001,0.025$, and 0.001 , respectively). The subset of triple-negative breast cancers (in other words, those with negative ER, PR, and HER-2/neu status) was positively associated with the methylation of the RIL/CDH13 panel and negatively associated with the HIN-1/RASSF1A panel. Mutations of $p 53$ were found in nine breast tumors (11\%), seven of which lacked methylation in both panels.

Conclusion We have defined two panels (HIN-1/RASSFIA, and $R / L / C D H 13)$ of methylation profiles, which correlated, either positively or negatively, to HR status.

\section{Introduction}

Over the past ten years, aberrant DNA methylation has been recognized as one of the most common molecular abnormalities in breast cancer $[1,2]$. A large body of evidence implicates potential hypermethylation of CpG islands in the loss of expression of a variety of crucial genes. Tumor-suppressor genes with aberrant methylation in breast cancers include
ARHI [3,4], RASSF1A [5], HIN-1 [6], the retinoic acid receptor II gene (RARß2) [7], hMLH1 [8], 14-3-3 $\sigma$ [9], RIZ1 [10], p16 [11], the E-cadherin gene [12], PTEN [13], and BRCA1 [14]. Methylation in breast cancer has been related to clinical and pathologic characteristics evident at presentation and clinical outcomes. A higher prevalence of $H I N-1$ and $R A R \beta 2$ methylation was found in the lymph nodes, bone, brain, and 
lung metastases than the primary tumor [15]. Widschwendter and colleagues [16] reported that the methylation of certain genes was associated with hormone receptor (HR) status, in addition to the response to treatment with tamoxifen. $A$ high prevalence of $P G R, H S D 17 B 4$, and $C D H 13$ methylation has been associated with HER-2/neu-positive breast cancer [17].

Methylation-specific PCR (MSP) is a sensitive assay used to detect methylation and analyze the methylation status of genes of interest. However, problems inherent to this assay (such as those caused by the use of different primers for the methylated or unmethylated alleles, the gel-based data analysis system used, and difficulties in quantitation) have caused frequent false-positive results in tissue-sample analyses. A new technique, pyrosequencing, has been adapted for use in highly sensitive and quantitative methylation analyses $[18,19]$. Pyrosequencing methylation analysis is a modification of the combined bisulfite restriction analysis (COBRA) that compares favorably with COBRA in sensitivity, specificity, and robustness [18]. Tost and colleagues also confirmed that the pyrosequencing technique is quantitative, amenable to the analysis of bisulfite-treated DNA derived from paraffin-embedded tissue samples, highly reproducible, and accurate [19]. Bisulfite pyrosequencing has been used in clinical trials of hypomethylating drug treatment and provides accurate and reliable results [20].

To investigate methylation profiles in breast cancer cells, we used bisulfite pyrosequencing to screen 12 known tumor-suppressor genes in 90 pairs of breast cancers and normal tissues. Although all 12 genes had been reported to exhibit hypermethylation in a fraction of breast cancer cases, our assays provided a comprehensive survey of their methylation status and confirmed that five genes could be useful in defining a methylation profile in breast cancer cells. Our findings also suggest that two panels of methylation profiles correlated, either positively or negatively, to HR status.

\section{Materials and methods Cell lines}

Human breast cancer cell lines SKBr3, MDA-MB-435, MDAMB-468, BT-20, MDA-MB-231, and MCF-7 were maintained in RPMI 1640 medium supplemented with 10\% fetal bovine serum. Normal breast epithelial cells, HMEC 231 and HMEC234, were cultured in a 1:1 solution of MCDB 105 and medium 199 with $15 \%$ fetal bovine serum and $10 \mathrm{ng} / \mathrm{ml}$ epithelial growth factor (Sigma, St Louis, MO, USA), as described elsewhere [21].

\section{Tissue samples}

We used 90 samples, consisting of paired tissues and associated clinicopathologic data from the Breast Tumor Bank at The University of Texas MD Anderson Cancer Center (Houston, TX, USA). The samples of breast tumors and corresponding adjacent normal-appearing tissues (from tissues located at least $3 \mathrm{~cm}$ away from the site at which the tumor was sampled) came from 80 patients who had undergone surgery at The University of Texas MD Anderson Cancer Center in 2004 or 2005 and 10 patients who had been diagnosed with breast cancer between 1995 and 2003. All tissue samples had been fresh-frozen and stored at $-80^{\circ} \mathrm{C}$. No patient was recruited specifically for this study. All patients gave written informed consent permitting the use of their breast tissue for research at the time specimens were collected. This study was approved by The University of Texas MD Anderson Cancer Center's Institutional Review Board.

\section{Histopathologic analysis}

All breast specimens were reviewed by experienced pathologists. Slides were subject to immunoperoxidase staining for estrogen receptors (ERs; clone 6F11, Novocastra Laboratories Ltd, Benton Lane, UK) and progesterone receptors (PRs; clone1A6, Novocastra Laboratories Ltd), according to the manufacturer's recommendations. Cancers were considered receptor-positive if $>10 \%$ of malignant cells showed nuclear staining. Cancers were classified as HR-positive if the ER and/ or PR status was positive. HER-2/neu gene amplification was assessed by interphase fluorescence in situ hybridization (FISH) using the PathVysion HER-2/neu probe kit (Vysis Inc., Downers Grove, IL, USA), according to the manufacturer's recommendations. Levels of the HER-2/neu:CEP17 signal ratio were considered normal if $\mathrm{FISH}$ detected $\leq 2.0$ copies per cell.

\section{Microdissection, DNA extraction, and sodium bisulfite treatment}

To avoid contamination with normal tissues in the methylation analysis, we isolated breast cancer cells and paired normal breast epithelial cells from tissues by manual microdissection, following the National Cancer Institutes (Bethesda, MA, USA) protocols [22]. In brief, 5-10 $\mu \mathrm{m}$ sections were cut from each archival fresh-frozen tissue block. For each pair of tissues, the presence of tumor cells in malignant tissues and absence of cancer cells in normal tissues were confirmed by histopathologic examination. Frozen tissue sections were fixed with ethanol, stained with $\mathrm{H} \& \mathrm{E}$, and microdissected using a needle. Each tumor sample contained $>70 \%$ tumor cells after microdissection. Genomic DNA was extracted from patient samples, breast cancer cell lines, and normal breast epithelial cells using the Dneasy tissue kit (Qiagen, Valencia, CA, USA). Bisulfite treatment of 1-2 $\mu \mathrm{g}$ of genomic DNA was performed, as previously described [3].

\section{Genes studied}

Twelve tumor-suppressor genes were selected for this study. $A R H I$ (CpG I and II), RASSF1A, HIN-1 (SCGB3A1), RAR 2 , $h M L H 1$, the 14-3-3 $\sigma$ gene, RIZ1, p16 (CDKN2A), and the Ecadherin gene $(C D H 1)$ were selected on the basis of previous reports of elevated methylation rates in breast cancers [3-12]. RIL (PDLIM4) [23], CDH13, and NKD2 were identified as 
hypermethylated genes using genome-wide methylated $\mathrm{CpG}$ island amplification (MCA) from multiple tumors (24). Global methylation was estimated by testing methylation levels of LINE1 repetitive elements [25]. We also assessed the methylation of $E R \alpha$ and $P G R B$ genes.

\section{Pyrosequencing methylation analysis}

Bisulfite pyrosequencing was used to detect methylation of all 15 genes. Pyrosequencing primers were designed using Assay Design software 1.0 (Biotage, Westborough, MA, USA). For each gene, we selected the $\mathrm{CpG}$ island region flanking the transcription start site at the 5'UTR. Two to six $\mathrm{CpG}$ sites were studied for each particular $\mathrm{CpG}$ island. The primers for pyrosequencing and PCR conditions are listed in Additional file 1. Bisulfite-treated DNA $(1 \mu \mathrm{l})$ was amplified in $50 \mu$ of reaction mixture, containing primers and $0.2 \mathrm{U}$ of Taq polymerase (New England Biolabs, Ipswich, MA, USA). For the amplification of $H I N-1, R A R \beta 2, h M L H 1$, the 14-3-3 $\sigma$ gene, $R I Z 1$, the E-cadherin gene, NKD2, and $P G R B$, we used a universal primer approach [18]. The PCR product was purified and methylation was quantitated using the PSQ HS 96A pyrosequencing system and Pyro gold reagents (Biotage). Methylation data are presented as the percentage of average methylation in all observed CpG sites. To set the controls for pyrosequencing, we used cancer cell lines and normal cells that were consistently positive or negative with stable levels of methylation. In this study, each PCR assay included a positive control (the MDA-MB-231 breast cancer cell line, which is highly methylated in most genes) and a negative control (normal breast epithelial cells, HMEC231, which are unmethylated in all genes, except LINE1, ARHI, and the 14-3-3 $\sigma$ gene). $\mathrm{RKO}$, a colon cancer cell line that is highly methylated in $h M L H 1$ and $p 16$, was also used as a positive control.

\section{p53 mutation detection}

Genomic DNA was extracted from microdissected tumor tissue using the QIAGEN DNA purification kit (Qiagen). PCR was performed using primers that amplify $p 53$ exons 5-6 and 7-9. The PCR products were then purified by the gel purification system (Qiagen) or Exonuclease I and shrimp alkaline phosphatase (USB, Cleveland, $\mathrm{OH}, \mathrm{USA}$ ). Mutations were determined by direct sequencing.

\section{Statistical methods and analysis}

In the studied cohort, adjacent normal breast tissue was taken from each of 90 patients during surgery. Taking advantage of paired normal/tumor samples in this study, we chose the value of normal samples as the reference. If using the sample mean plus two times the standard deviation of the pooled normal samples (and a minimum of $10 \%$ methylation) as a cut-off point, there is $>97 \%$ probability that the methylation level for a normal tissue will be lower than the cut-off point. It is reasonable to assume that a value larger than the cut-off point is likely to be abnormal (or positive). Panels of genes, that is to say, $H I N-1 / R A S S F 1 A$ and RILICDH13 were considered positive if both markers in each panel were positive. Descriptive analyses were performed first for exploratory purposes. Pair-wise scatter plots are presented to show the correlations among the genes methylated. Heat maps were plotted to show levels of gene expression or methylation using hierarchical clustering to visually represent the association of different genes or samples by histopathologic tumor characteristics (ERs, PRs, and HER-2/neu). Chi-square or Fisher's exact test were used to assess the dependence between two categorical variables. Pearson's correlation coefficient was used to assess the relationship between two continuous variables. The Wilcoxon rank-sum test was used to compare either continuous or categorical variables between two groups. Analysis of variance (ANOVA) was applied to compare the values of gene methylation with tumor characteristics. Multivariate logistic regression was used to assess the ability of various levels of gene methylation to predict the ER, PR, HR, or HER-2/neu status. All reported $p$ values are two-sided and considered statistically significant if $p<0.05$. Analyses were performed using $S$ PLUS 2000 software (Insightful Corp., Seattle, WA, USA).

\section{Results \\ Patients' clinical data}

Patient characteristics are presented in Table 1. Normal paired tissues were unavailable in two cases. Information regarding the grade, HER-2/neu status, and ER and PR status were unavailable for one, ten, and three patients, respectively.

\section{Accuracy, reproducibility, and quantitation of results with bisulfite pyrosequencing}

To confirm the reliability of bisulfite pyrosequencing for the quantitation of methylation levels, we measured the methylation of $A R H I \mathrm{CpG}$ islands I and II using pyrosequencing and COBRA and compared the resulting data. COBRA detected only one $\mathrm{CpG}$ site, whereas pyrosequencing detected four to eight $\mathrm{CpG}$ sites in one $\mathrm{CpG}$ island (Figure 1). From six breast cancer cell lines and two cultures of normal breast epithelial cells, methylation data were matched in $\mathrm{CpG}$ island II but not completely matched in CpG island I. COBRA showed two breast cancer cell lines were partially methylated in $\mathrm{CpG}$ island I (50\% and 54\% in one CpG site), but they were shown to be hypermethylated in $\mathrm{CpG}$ island I by pyrosequencing (the mean of four CpG sites per island was $92 \%$ and $91 \%$, respectively). On the basis of these results, pyrosequencing was more sensitive for methylation detection than COBRA.

To confirm the reproducibility of pyrosequencing, the methylation of $H I N-1$ and $R / L$ was measured repeatedly in 22 samples using bisulfite treatment and pyrosequencing assays. The correlation between repeated and initial values was high (for HIN1 , Pearson's correlation coefficient $R=0.99$ and $p<0.0001$; for $R I L, R=0.99$ and $p<0.0001)$. In addition, the variation between individual assays was $<6 \%$ : the mean differences and $95 \%$ confidence intervals (Cls) for HIN-1 and RIL were 
Table 1

\section{Patient characteristics}

Clinicopathologic factors Number of sample

Age

90

Median, range

57 years, 29-81 years

Tumor grade (BNG)

89

3

47

3

39

Tumor size

90

$\mathrm{T} 1$

19

T2

T3

T4

Stage

90

I

॥

III

Lymph-node metastasis

pNO

$\mathrm{pN} 1$

$\mathrm{pN} 2$

$\mathrm{pN} 3$

Lymphatic invasion

Positive

Page 4 of 13

(page number not for citation purposes)
Table 1 (Continued)

Patient characteristics

Negative

45

Vascular invasion

78

Positive

32

Negative

46

ER status

87

Positive

52

Negative

PR status

87

Positive

40

Negative

47

Hormone receptor status

87

Positive (ER- and/or PR-positive)

55

Negative (both ER- and PR-negative)

32

HER-2/neu status

80

Positive

Negative

73

Histology

90

Ductal

62

Lobular

8

Mixed ductal and lobular

9

Other

11

BNG, Black's nuclear grade; ER, estrogen receptor; PR, progesterone receptor. 


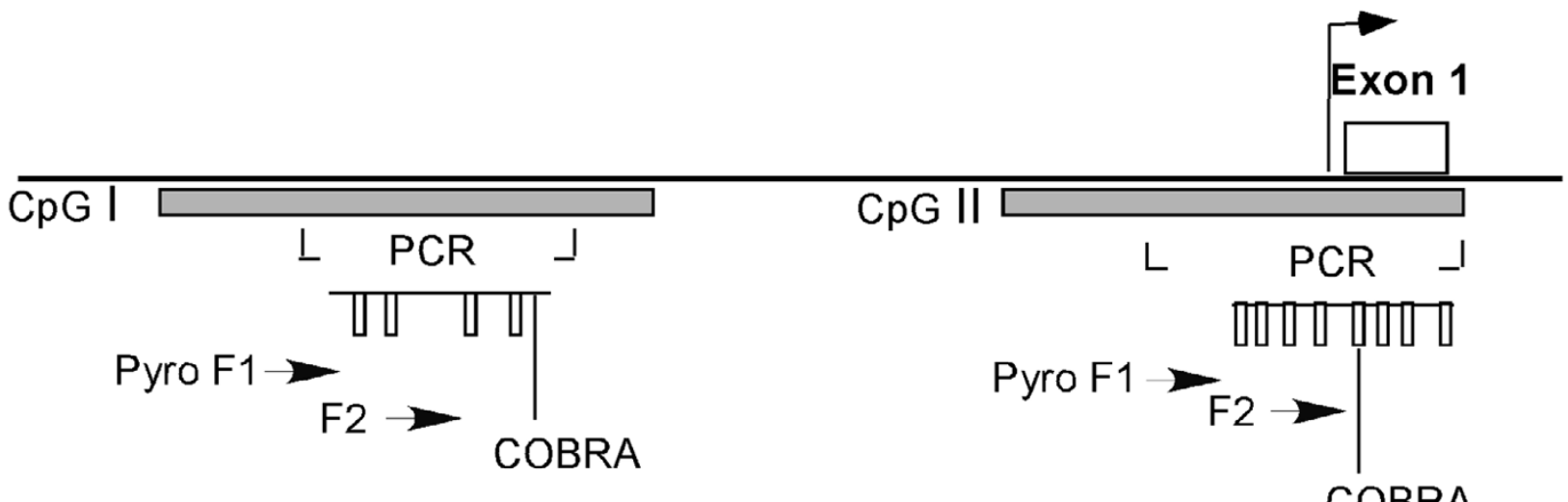

B

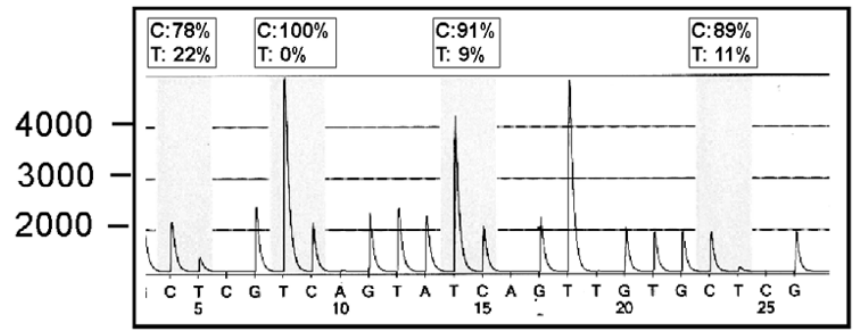

CpG island II (Pyro F1)

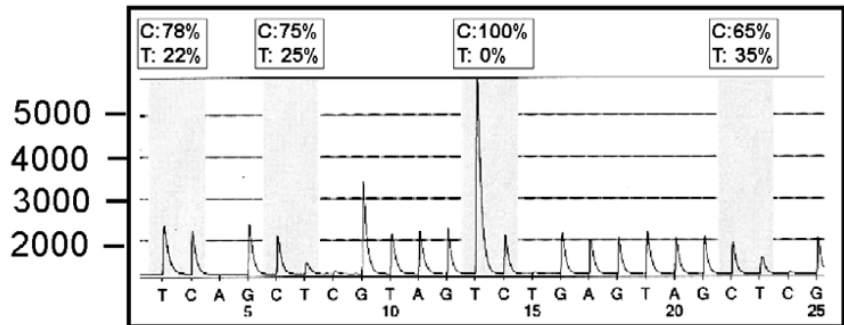

CpG island II (Pyro F2)

Pyrosequencing analysis for $A R H /$ methylation. (a) Design of pyrosequencing primers for $A R H I \mathrm{CpG}$ islands I and II. The vertical bars indicate CpG sites in PCR products. COBRA indicated the restriction enzyme cutting site. (b) Pyrosequencing results for ARHI CpG island II methylation in MBMDA-231 cells using two detection primers (Pyro F1 and F2). Methylation levels ranged from 65\% to 100\%, with a mean of $84 \%$. Gray highlighting marks the $\mathrm{CpG}$ sites tested and the percentage reading of $\mathrm{C}$ and T. COBRA, combined bisulfite restriction analysis.

$1.9 \%$ with $95 \%$ Cls of $-0.1 \%$ to $3.9 \%$ and $3.0 \%$ with $95 \%$ Cls of $0.1 \%$ to $6.0 \%$, respectively.

\section{Frequent methylation of RIL, HIN-1, RASSF1A, CDH13, and $\boldsymbol{R A R} \beta \mathbf{2}$}

We evaluated the methylation status of 12 putative tumor-suppressor genes (ARHI, RASSF1A, HIN-1, RAR $32, h M L H 1$, the 14-3-3 $\sigma$ gene, $R I Z 1, p 16$, the E-cadherin gene, $R I L, C D H 13$, and NKD2), LINE1,ER, and PGRB in both culture cell lines and primary cancer tissues. The results from the analysis of six breast cancer cell lines and two normal breast epithelial cell samples (Additional file 2) indicated that 7 of 12 genes (RIL, HIN-1, RASSF1A, ARHI, CDH13, RAR 32 , and NKD2) were densely methylated (marked bold) in cancer cell lines but not in normal breast epithelial cell lines. We also tested the methylation profiles of the 12 genes in breast cancer and the adjacent normal breast tissues from the same patients. In our pilot study, we screened 12 tumor-suppressor genes in 32-37 paired tissues. Five tumor-suppressor genes (RASSF1A, RIL, $H I N-1, C D H 13$, and $R A R \beta 2)$ were frequently methylated in breast cancer tissues (the positive rate ranged from $17 \%$ to $58 \%$ ) but not in normal breast tissues (the positive rate ranged from $0 \%$ to $4.5 \%)$. Another five genes ( $R / Z 1$, the E-cadherin gene, $p 16, h M L H 1$, and NKD2) were not frequently methylated in either malignant or normal breast tissue (the positive rate ranged from $0 \%$ to $9 \%$, all $<10 \%$ ). The $14-3-3 \sigma$ gene is highly methylated in both malignant and normal breast tissues. $A R H I$, as an imprinted gene, is at least partially methylated in all cases (Figure 2 and Table 2). Consequently, seven genes were unsuitable for development of methylation profiles. To correlate the methylation profile to clinical outcomes, we focused on the five highly methylated genes by assaying 53 more paired samples. $P G R B$ was not frequently methylated in the 48 cases of breast tumor or normal breast tissue. ER was not highly methylated in the 40 cases of breast tumor or normal breast tissue, but higher methylation levels were observed in normal tissues. LINE1, a global methylation marker, was highly methylated in all normal and malignant tissues, but the methylation levels were significant lower in tumors $(60.0 \pm$ 8.8) than normal tissues $(68.2 \pm 3.5 ; p<0.001)$. 
Table 2

Methylation levels of $\mathbf{1 5}$ genes from pairs of normal and malignant breast tissues

\begin{tabular}{|c|c|c|c|c|}
\hline \multirow[t]{2}{*}{ Gene } & \multirow[t]{2}{*}{ Number of cases studied } & \multicolumn{3}{|c|}{ Methylation level (mean \pm SD) (Positive rate*) } \\
\hline & & Normal & Cancer & pvalue $^{* *}$ \\
\hline LINE1 & 90 & $68 \pm 4$ & $60 \pm 9$ & $<0.001$ \\
\hline$R / L$ & 90 & $10 \pm 4(4.5)$ & $27 \pm 22(57)$ & $<0.001$ \\
\hline$H I N-1$ & 90 & $5 \pm 3(2.3)$ & $20 \pm 20(49)$ & $<0.001$ \\
\hline RASFF1A & 90 & $3 \pm 3(3.4)$ & $19 \pm 20(58)$ & $<0.001$ \\
\hline $\mathrm{CDH} 13$ & 90 & $3 \pm 2(0)$ & $14 \pm 13(44)$ & $<0.001$ \\
\hline$R A R \beta 2$ & 90 & $2 \pm 1(0)$ & $7 \pm 12(17)$ & $<0.001$ \\
\hline ARHI CpG I & 32 & $30 \pm 6(0)$ & $47 \pm 16(49)$ & $<0.001$ \\
\hline$A R H I \mathrm{CpG} I I$ & 32 & $38 \pm 7(2)$ & $36 \pm 10(2)$ & 0.250 \\
\hline$R I Z 1$ & 37 & $2 \pm 2(0)$ & $3 \pm 4(5)$ & 0.111 \\
\hline E-cadherin & 34 & $4 \pm 3$ (3) & $4 \pm 4(9)$ & 0.163 \\
\hline p16 & 33 & $2 \pm 1(0)$ & $1 \pm 1(0)$ & 0.044 \\
\hline$h M L H 1$ & 33 & $2 \pm 3(6)$ & $1 \pm 1(0)$ & 0.864 \\
\hline $14-3-3 \sigma$ & 10 & $75 \pm 21$ & $60 \pm 28$ & 0.275 \\
\hline$N K D 2$ & 47 & $2 \pm 4(2)$ & $4 \pm 7$ (8.5) & 0.308 \\
\hline$P G R B$ & 48 & $3 \pm 2(0)$ & $2 \pm 3(0)$ & 0.594 \\
\hline$E R \alpha$ & 40 & $7 \pm 4$ (3) & $5 \pm 3(5)$ & $<0.001$ \\
\hline
\end{tabular}

*Positive rate using the sample mean plus two times the SD of the pooled normal samples (and minimum 10\% methylation) as a cut-off point.

${ }^{\star \star} p$ value computed using the Wilcoxon signed rank test for paired data. SD, standard deviation.

\section{Correlation of methylation between different genes and to age}

On the basis of data from continuous marker methylation analyses in normal tissues, the methylation levels of RASSF1A and $H I N-1$ exhibited a strong correlation to each other $(R=0.66$, $p<0.001)$. Some moderate correlations were found between $R A R \beta 2$ and $C D H 13(R=0.40, p<0.001)$ and between $R I L$ and $C D H 13(R=0.30, p=0.004)$. Similarly, in tumor tissues, a moderate correlation existed between RASSF1A and HIN-1 $(R=0.51, p<0.001)$ and a weak correlation existed between $R I L$ and $C D H 13(R=0.19, p=0.068)$. In addition, the global methylation marker $L I N E 1$ was negatively correlated to $R I L$ ( $R$ $=-0.25, p=0.019)$ and RASSF1A $(R=-0.26, p=0.014)$ in breast cancer samples.

In normal tissues, $R / L$ methylation levels increased with age $(R$ $=0.336, p=0.001$ ), whereas other markers had little correlation to age. In the cancer tissues, HIN-1 levels exhibited a weak correlation to age $(R=0.229, p=0.03)$, whereas other markers had little correlation to age.

\section{Correlation between methylation in malignant and adjacent normal tissues}

To study whether gene methylation in cancer would affect adjacent normal-appearing mammary gland tissues, the pairwise correlation between paired tumor and adjacent normal breast tissues was estimated for each marker. Methylation of RIL, HIN-1, RASSF1A, and CDH13 exhibited a positive correlation between breast cancer and normal tissues. The Pearson correlation values ranged from 0.25 to 0.38 ( $p$ values ranged from 0.02 to 0.0002 ).

\section{Relationship between methylation of individual genes and clinical characteristics}

Using the ANOVA model, no statistically significant associations were found between clinical stage, tumor size, or node status. Tumors that were poorly differentiated (grade 3) according to Black's nuclear grade (BNG) had lower methylation levels of $H I N-1$ than well- and moderately differentiated tumors (grade $1+2 ; p=0.01$ ). Similarly, RASSF1A methylation levels were lower in poorly differentiated cancers than well- and moderately differentiated tumors ( $p=0.053)$. In addition, $\mathrm{CDH} 13$ and $\mathrm{RIL}$ methylation levels cancers were marginally higher in poorly differentiated than well- and moderately differentiated cancers $(p=0.059$ and 0.096, respectively).

\section{Relationship between methylation of individual genes} and ER, PR, HR, and HER-2/neu status

In univariate analyses, the ER status was positively associated with high $H I N-1$ and RASSF1A methylation levels but negatively correlated to high $R / L$ methylation levels $(p<0.001$, 
Figure 2
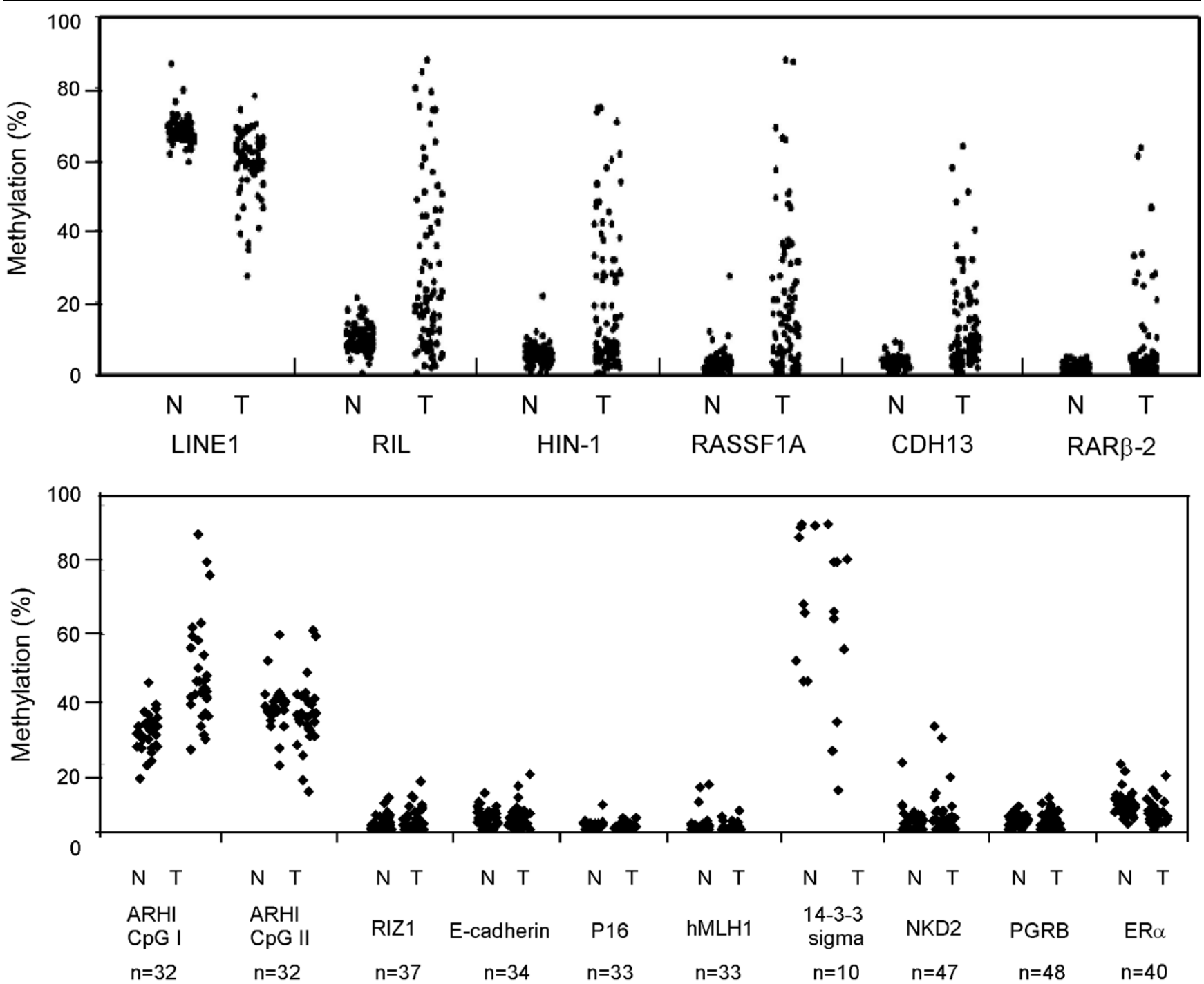

Comparison of promoter methylation levels of 15 genes in paired normal and malignant breast tissue samples.

0.002, and 0.01, respectively). Specifically, patients with a positive ER status had higher methylation levels of $H I N-1$ (mean of 29\% in ER-positive tumors versus 9\% in ER-negative tumors), whereas patients with a negative ER status had higher methylation levels of RIL (mean of 36\% in ER-negative tumors versus $22 \%$ in ER-positive tumors). In addition, the PR status was positively associated with high $H I N-1$ methylation levels $(p<0.001)$ and negatively associated with high $\mathrm{CDH} 13$ methylation levels $(p=0.03)$. By defining the HR status as positive if the status of ERs and/or PRs is positive, the associations between the methylation markers and HRs are similar to those between the methylation markers and ERs. In multivariate logistic models, high levels of both $H I N-1$ and RIL methylation are independent predictors of the status of ERs and HRs, and high levels of HIN-1 and $\mathrm{CDH} 13$ methylation are independent predictors of PR status (Table 3 ). Higher methylation levels of $H I N-1$ were more likely to be associated with
ER-positive than ER-negative tumors, whereas higher methylation levels of $R / L$ were more likely to be associated with ERnegative than ER-positive tumors. Using multivariable logistic models, we have assessed the effect of each gene (LINE1, RIL, HIN-1, RASFF1A, CDH13, and RAR 32 ) on HR (adjusted for age), ER (adjusted for age and PR status), or PR (adjusted for age and ER status) status (Additional file 3 ). The results shown in Additional file 3 are similar to those in Table 3. Among the markers studied, the higher methylation levels of $H I N-1$ were significantly correlated to a lack of HER-2/neu amplification $(p=0.014)$.

\section{Correlation of two panels of methylation profiles to opposite hormone status}

Because of a correlation between the methylation levels of several of the genes studied, we assembled four potential markers into two panels: HIN-1/RASSFIA and RIL/CDH13. A 
Table 3

Fitted multivariate logistic models for ER, PR, and HR expression

\begin{tabular}{|c|c|c|c|c|c|}
\hline \multirow[t]{2}{*}{ Single gene model } & \multicolumn{5}{|c|}{ Two-panels model } \\
\hline & Odds ratio $(95 \% \mathrm{Cl})$ & pvalue & & Odds ratio $(95 \% \mathrm{Cl})$ & pvalue \\
\hline ER & & & $\mathrm{ER}$ & & \\
\hline$H I N-1\left(\times 10^{-1}\right)$ & 0.399 (0.25 to 0.63$)$ & 0.0001 & HIN-1/RASSF1A & 0.06 (0.01 to 0.25$)$ & $<0.0001$ \\
\hline$R / L\left(\times 10^{-1}\right)$ & 1.525 (1.11 to 2.10$)$ & 0.0094 & $C D H 13 / R / L$ & 10.14 (2.65 to 38.76$)$ & 0.001 \\
\hline PR & & & PR & & \\
\hline$H I N-1\left(\times 10^{-1}\right)$ & 0.551 (0.41 to 0.74$)$ & 0.0001 & HIN-1/RASSF1A & 0.17 (0.06 to 0.46$)$ & $<0.0001$ \\
\hline $\mathrm{CDH} 13\left(\times 10^{-1}\right)$ & 1.674 (1.05 to 2.67$)$ & 0.0305 & $C D H 13 / R / L$ & 3.51 (1.17 to 10.51$)$ & 0.025 \\
\hline HR & & & $\mathrm{HR}$ & & \\
\hline$H I N-1\left(\times 10^{-1}\right)$ & 0.319 (0.18 to 0.57$)$ & 0.0001 & HIN-1/RASSF1A & 0.05 (0.01 to 0.23 ) & $<0.0001$ \\
\hline$R / L\left(\times 10^{-1}\right)$ & 1.481 (1.08 to 2.03$)$ & 0.0153 & $C D H 13 / R / L$ & $10.18(2.67$ to 38.81$)$ & 0.001 \\
\hline
\end{tabular}

$\mathrm{Cl}$, confidence interval; ER, estrogen receptor; $\mathrm{HR}$, hormone receptor; $\mathrm{PR}$, progesterone receptor.

univariate analysis was performed and a multivariate logistic model was fitted to the two panels. The results of the univariate analysis for each panel indicated that the panels had a greater power to predict ER, PR, and HR status (Table 4 and Figure 3) than the methylation of individual genes (Table 3 ). Methylation of the HIN-1/RASSFIA panel was strongly correlated to positive ER, PR, and HR expression; $88 \%$ of $H I N-1 /$ RASSFIA-positive breast cancers were ER-positive and only $12 \%$ of breast cancers were ER-negative $(p<0.001)$. Conversely, methylation of the RIL/CDH13 panel was strongly correlated to negative ER, PR, and HR expression, because $69 \%$ of methylation-positive tumors were ER-negative and only $31 \%$ were ER-positive $(p=0.001)$.

We summarize the fitted multivariate logistic models of the HIN-1/RASSF1A and RIL/CDH13 panels by categorical variables (Table 3). Specifically, a patient with a positive value in the RIL/CDH13 panel is ten times more likely to have an ERnegative tumor than a patient with a negative value. By contrast, a patient with a positive value in the HIN-1/RASSF1A panel is 17 times more likely to have an ER-positive tumor than a patient with a negative value.

Interestingly, we found that triple-negative breast cancers (in other words, those with negative ER, PR, and HER-2/neu status) were positively correlated to methylation of the R/L/ $\mathrm{CDH} 13$ panel and negatively correlated to methylation of the HIN-1/RASSFIA panel (Table 4). In particular, 58\% (15 out of 26) of triple-negative cancers exhibited methylation of the RIL/ CDH13 panel, whereas 17\% (11 out of 64 ) of triple-negative cancers failed to methylate in this panel $(p<0.001)$. Only $9 \%$ (3 out of 34 ) of triple-negative cancers exhibited HIN-1/ RASSFIA panel methylation, whereas $41 \%$ (23 out of 56 ) of triple-negative cancers failed to methylate in this panel ( $p=$ 0.001). In addition, triple-negative cancers were significantly associated with advanced tumor grade (24\% in grade 1 and 2 versus $76 \%$ in grade $3 ; p=0.001$ ).

Breast tumors with p53 mutations lacked methylation

We studied the mutation status of $p 53$ in the 84 tumors in which DNA sequences were available for analysis. Overall, p53 mutations were found in 9 out of 84 (11\%) cases. Seven cases with $p 53$ mutations belonged to the group that lacked methylation in both panels; one case was positive in the HIN1/RASSF1A panel and one case was positive in the RIL/ $\mathrm{CDH13}$ panel. In the methylation-negative group (both panels were negative), $18 \%$ (7 out of 38 ) of the specimens had $p 53$ mutations; in the methylation-positive group (either panel or both panels were positive), only 4\% (2 out of 46 ) of specimens had $p 53$ mutation ( $p=0.072$ in Fisher's exact test). Interestingly, five of the cases with $p 53$ mutations occurred in triplenegative (ER-, PR-, and HER2-negative) cancers.

\section{Discussion}

Although a large body of evidence has demonstrated that aberrant DNA methylation has an important role in breast carcinogenesis, variation in the data is still a problem. In this study, we used bisulfite pyrosequencing to quantitate the methylation of 12 known tumor-suppressor genes in breast cancers. To avoid contamination with normal tissues, we isolated breast cancer cells by microdissection and compared levels of methylation with those in normal breast epithelial cells from the same patients. Bisulfite pyrosequencing provided sensitive and reproducible measurements. The variation between the different assays was $<6 \%$. Our data confirmed that RIL, HIN-1, RASSF1A,CDH13, and RAR 22 were frequently methylated in breast cancers but not in normal breast tissues. The other six genes were not highly methylated in breast cancers or methylated in either malignant and normal breast tissues; $A R H I$, as an imprinted gene, has a different 
Univariate analysis of clinical variables by HIN-1/RASSF1A and RIL/CDH13panels, according to Fisher's exact test

\begin{tabular}{|c|c|c|c|c|c|c|}
\hline \multirow[b]{3}{*}{ Tumor size } & \multicolumn{3}{|c|}{ HIN-1/RASSF1A panel } & \multicolumn{3}{|c|}{ RIL/CDH13 panel } \\
\hline & $\begin{array}{l}\text { Number negative } \\
\text { (percentage) }\end{array}$ & $\begin{array}{c}\text { Number positive } \\
\text { (percentage) }\end{array}$ & $p$ value & $\begin{array}{l}\text { Number negative } \\
\text { (percentage) }\end{array}$ & $\begin{array}{l}\text { Number positive } \\
\text { (percentage) }\end{array}$ & $p$ value \\
\hline & & & 0.502 & & & 0.999 \\
\hline 1 & $11(20)$ & $8(24)$ & & $14(22)$ & $5(19)$ & \\
\hline 2 & $20(36)$ & $16(47)$ & & $25(39)$ & $11(42)$ & \\
\hline 3 & $12(21)$ & $6(18)$ & & $13(20)$ & $5(19)$ & \\
\hline 4 & $13(23)$ & $4(12)$ & & $12(19)$ & $5(19)$ & \\
\hline Tumor grade (BNG) & & & 0.015 & & & 0.162 \\
\hline $1+2$ & $25(45)$ & $25(74)$ & & $39(61)$ & $11(44)$ & \\
\hline 3 & $30(55)$ & $9(26)$ & & $25(39)$ & $14(56)$ & \\
\hline Tumor stage & & & 0.16 & & & 0.745 \\
\hline 1 & $8(14)$ & $7(21)$ & & $12(19)$ & $3(12)$ & \\
\hline 2 & $20(36)$ & $17(50)$ & & 25 (39) & $12(46)$ & \\
\hline 3 & $28(50)$ & $10(29)$ & & $27(42)$ & $11(42)$ & \\
\hline Lymph-node metastasis & & & 0.558 & & & 0.78 \\
\hline PNO & $24(43)$ & $15(44)$ & & $26(41)$ & $13(50)$ & \\
\hline PN1 & $16(29)$ & $12(35)$ & & $22(34)$ & $6(23)$ & \\
\hline PN2 & $5(9)$ & $4(12)$ & & $6(9)$ & $3(12)$ & \\
\hline PN3 & $11(20)$ & $3(9)$ & & $10(16)$ & $4(15)$ & \\
\hline Lymphatic invasion & & & 0.244 & & & 0.805 \\
\hline 1 & $25(52)$ & $20(67)$ & & $32(59)$ & $13(54)$ & \\
\hline 2 & $23(48)$ & $10(33)$ & & $22(41)$ & $11(46)$ & \\
\hline Vascular invasion & & & 0.346 & & & 0.623 \\
\hline 1 & $26(54)$ & $20(67)$ & & $33(61)$ & $13(54)$ & \\
\hline 2 & $22(46)$ & $10(33)$ & & $21(39)$ & $11(46)$ & \\
\hline ER status & & & $<0.001$ & & & 0.001 \\
\hline Positive & $22(42)$ & $30(88)$ & & $44(72)$ & $8(31)$ & \\
\hline Negative & $31(58)$ & $4(12)$ & & $17(28)$ & $18(69)$ & \\
\hline PR status & & & $<0.001$ & & & 0.033 \\
\hline Positive & $16(30)$ & $24(71)$ & & $33(54)$ & $7(27)$ & \\
\hline Negative & $37(70)$ & $10(29)$ & & $28(46)$ & $19(73)$ & \\
\hline HR status & & & $<0.001$ & & & 0.001 \\
\hline Positive & $24(45)$ & $31(91)$ & & $46(75)$ & $9(35)$ & \\
\hline Negative & $29(55)$ & $3(9)$ & & $15(25)$ & $17(65)$ & \\
\hline HER-2/neu status & & & 0.041 & & & 0.999 \\
\hline Positive & $7(14)$ & $0(0)$ & & $5(9)$ & $2(8)$ & \\
\hline Negative & $43(86)$ & $30(100)$ & & $50(91)$ & $23(92)$ & \\
\hline Triple-negative & & & 0.001 & & & $<0.001$ \\
\hline No & 33 (59) & $31(91)$ & & $53(83)$ & $11(42)$ & \\
\hline Yes & $23(41)$ & 3 (9) & & $11(17)$ & $15(58)$ & \\
\hline
\end{tabular}

BNG, Black's nuclear grade; ER, estrogen receptor; HR, hormone receptor; PR, progesterone receptor. 


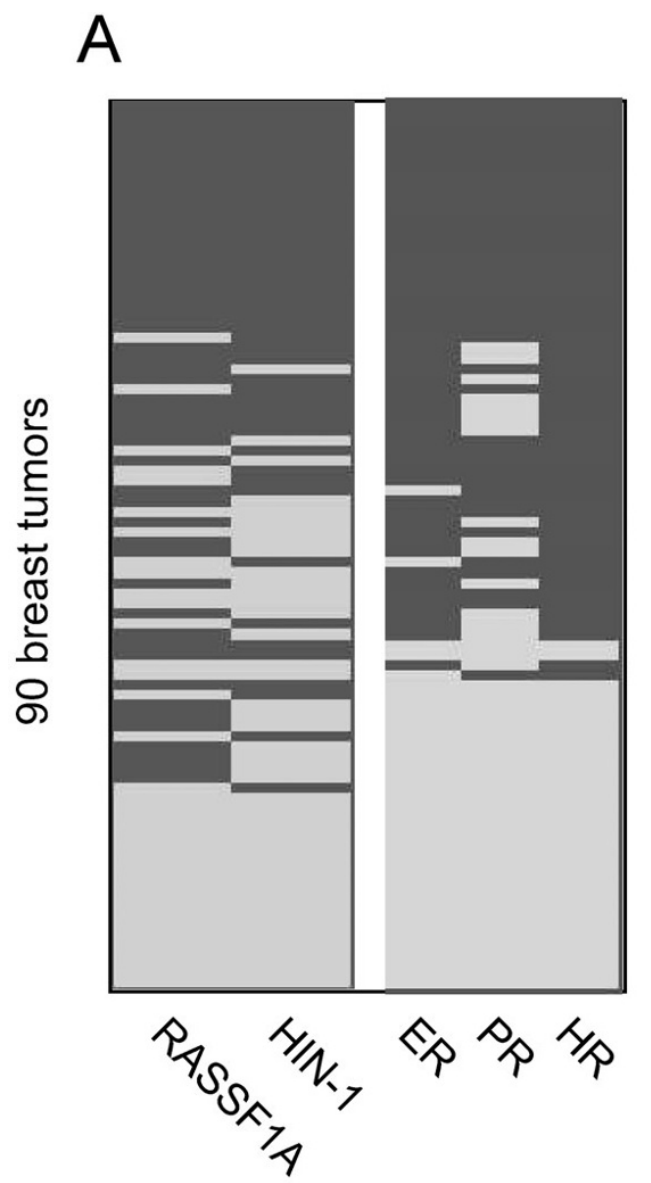

B

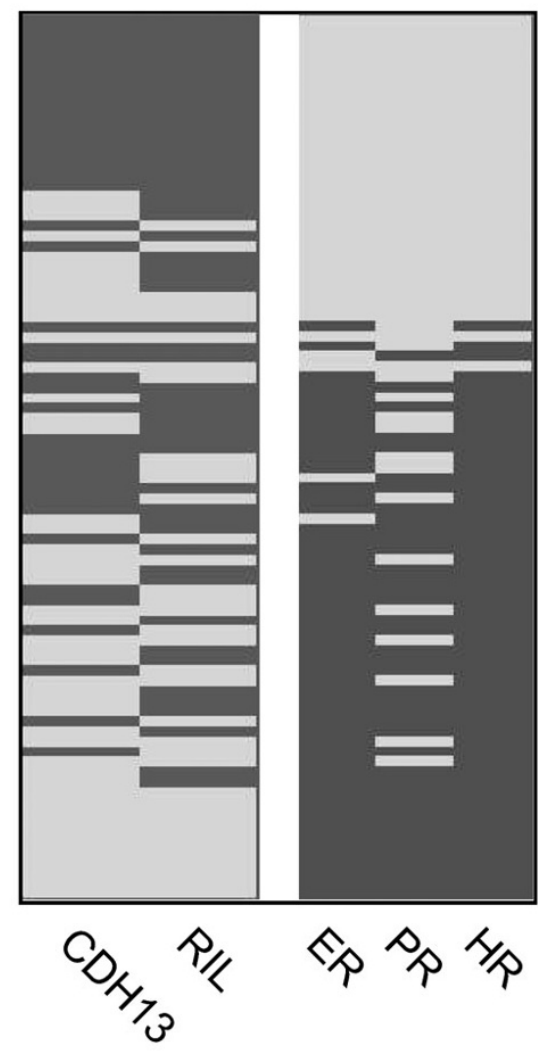

Comparison of two methylation panels with hormone receptor status. (a) Dichotomous heat map representing DNA methylation in the HIN-1/ RASSF1A panel (left) and hormone status of each tumor (right). (b) Dichotomous heat map representing DNA methylation data in the RIL/CDH13 panel (left) and hormone receptor status of each tumor (right). Dark gray, positive; light gray, negative.

methylation status. All the inconsistencies indicate that these seven genes are unsuitable for methylation profile studies.

In the past, methylation data have been correlated to clinical pathologic parameters, to clarify the role of methylation in breast carcinogenesis. Our most interesting finding was a correlation between gene methylation and the HR status. Methylation in breast cancer has already been connected to hormone regulation, but the correlation is not clear yet. Campan and colleagues [26] reviewed the DNA methylation profiles of breast, endometrial, ovarian, and proximal colon cancers but did not find evidence for global hormone-specific DNA methylation alterations. Widschwendter and colleagues [16] reported significant differences in the HR status between clusters of DNA methylation profiles. Their results suggested the existence of an interaction between DNA methylation and HR biology in breast cancer cells. In our own study, we found that the ER status was positively associated with the methylation of $H I N-1$ and RASSF1A but negatively correlated to the methylation of $R / L$. In addition, the PR status was positively associated with the methylation of $H I N-1$ and negatively associated with the methylation of $\mathrm{CDH} 13$. Moreover, if data from the methylation of individual genes were combined into two panels, methylation of the HIN-1/RASSF1A panel strongly predicted the expression of ERs, PRs, and HRs and methylation of the RIL/ $\mathrm{CDH} 13$ panel strongly predicted the negative expression of ERs, PRs, and HRs.

The status of ERs, PRs, and HRs has been recognized as an important prognostic factor in patients with breast cancer, in addition to a predictive marker for the response to treatment with endocrine therapy. The presence of ERs and/or PRs is predictive of the response to treatment with the antiestrogen tamoxifen [27]. ER and PR expression patterns are heavily influenced by changes in the chromatin structure during transcription. Indeed, both the predominant mammalian DNA methyltransferase and histone deacetylases have crucial roles in maintaining transcriptionally repressive chromatin by forming suppressive complexes at replication foci [28]. Our current 
studies provide evidence that epigenetic changes are tightly connected with HR regulation in breast cancer.

Interestingly, ERs and PRs were not frequently methylated in breast cancers at levels comparable with those observed in the five tumor-suppressor genes. Consequently, alterations in methylation do not seem to have silenced these receptors directly. Increased methylation of certain genes was associated with the expression of ERs and PRs, suggesting that silencing of, at least some, tumor suppressors might affect the transcriptional regulation of $\mathrm{HRs}$, possibly by upregulating $\mathrm{HR}$ co-stimulators. Conversely, the downregulation of ERs or PRs might relate to the increased expression of HR co-repressors. These hypotheses will be tested in future studies.

Recently, on the basis of the microarray profiling of invasive breast carcinomas, five distinct subtypes of tumors (luminal A, luminal $B$, normal breast-like, HER-2/neu overexpressing, and basal) associated with different clinical outcomes have been identified $[29,30]$. The basal subtype is associated with poor clinical outcomes and the subtype observed in BRCA1related breast cancers. All basal-like tumors tested in the current study were triple-negative (that is to say, negative for ER, $\mathrm{PR}$, and HER-2/neu expression), poorly differentiated, and high-grade $[29,30]$. An early study of HIN-1 methylation revealed lower frequencies of $H I N-1$ promoter methylation in sporadic breast tumors with a 'BRCA1-like' histopathologic phenotype [31]. In the 90 cases we tested, $76 \%$ of the 26 triple-negative tumors were high-grade. Interestingly, we found these tumors to be positively associated with methylation of the RIL/CDH13 panel but negatively correlated to methylation of the HIN-1/RASSFIA panel. Our results suggested that methylation reflected by the RIL/CDH13 panel might have a role in the phenotype of basal-like breast tumors.

Recent advances in molecular biology have revealed numerous genetic alterations involved in breast tumorigenesis; $p 53$ mutation is among the most important of those alterations, and studies have reported that $p 53$ mutations are strongly associated with poor prognoses in breast cancer [32]. In colorectal cancer, methylation phenotypes define two groups with significantly different genetic lesions (K-RAS and p53 mutations) [33]. In this study, we have identified $11 \%$ of breast tumors that had $p 53$ mutations. Equivalent to the observation in colorectal cancers, most cases with $p 53$ mutations belong to the group that lack methylation, suggesting that $p 53$ mutation and methylation can be two distinct mechanisms that deactivate tumor-suppressor genes in breast cancer. The correlation of $p 53$ mutation to hypomethylation is also likely to be owing to the fact that $p 53$ mutations and hypomethylation both occur in basal-like triple-negative breast cancers.

The methylation of multiple tumor-suppressor genes has been correlated to poor prognoses in cancers [34]. The use of epigenetic information has shown promise in the identification of patients with gastrointestinal cancers who have poor prognoses [35]. In esophageal carcinoma, the cancers with frequent methylation had significantly poorer survival, and methylation was a better predictor of outcome than the disease stage or patient age [36]. Methylation, as a prognosis factor, has also been described in bladder cancer [37], head and neck cancer [38], ovarian cancer [39], and acute lymphocytic leukemia [40]. In our study, we did not have sufficient survival data to correlate methylation to prognosis (most patients were treated in 2004 and 2005), but we did confirm that the methylation of multiple tumor-suppressor genes was an early event in the subgroups of patients with breast cancer. To understand whether methylation is a prognostic factor in breast cancer, we must conduct studies with longer follow-up times to obtain adequate survival data.

Finally, epigenetic therapy, including the use of demethylating agents (for example, 5-aza-cytidine and 5-aza-2'-deoxycytidine) and histone deacetylase inhibitors (for example, suberoylanilide hydroxamic acid and valproic acid), is currently in clinical trials for myelodysplastic syndrome, leukemia, ovarian cancer, and lung cancer. Previous reports indicate that the 'cross-talk' between inhibitors of DNA methylation and histone deacetylase can result in synergistic activation of silent tumorsuppressor genes in breast cancer. These observations suggest that combination of these inhibitors might be an effective form of epigenetic therapy for breast cancer. It is possible that epigenetic therapy will have a role in the management of breast cancer. The information from this study of methylation profiles will be useful for the study of the biology of breast cancer and refining epigenetic therapy.

\section{Conclusion}

By pyrosequencing methylation analysis, we have examined the methylation profile of 90 normal/breast cancer paired samples. Our data indicated that 5 out of 12 tumor-suppressor genes were frequently methylated in breast cancers but not the normal breast. We have defined two panels $(H I N-1)$ RASSFIA and RIL/CDH13) of methylation profiles, which correlated, either positively or negatively, to HR status.

\section{Competing interests}

The authors declare that they have no competing interests.

\section{Authors' contributions}

WF, LS, XH, and JJ carried out the methylation assays and helped prepare the manuscript. $\mathrm{SH}$ and $\mathrm{MH}$ carried out the p53 mutation assays. SW and YS performed the statistical analysis. AAS and $\mathrm{KKH}$ provided tissues and clinical data. DGR and JL provided pathologic supports for tissue microdisection. $Y Y, J$, and RCB conceived the study and prepared the manuscript. All authors read and approved the final manuscript. 


\section{Additional files}

The following Additional files are available online:

\section{Additional file 1}

Primers and PCR conditions for pyrosequencing assays. See http://www.biomedcentral.com/content/

supplementary/bcr1762-S1.doc

\section{Additional file 2}

Methylation levels of 12 tumor-suppressor genes, ER $\alpha$, PGRB, and LINE1 in six breast cancer cell lines and two normal breast epithelial cell cultures.

See http://www.biomedcentral.com/content/

supplementary/bcr1762-S2.doc

\section{Additional file 3}

Multivariable logistic models for assessing effect of each gene (LINE1, RIL, HIN-1, RASFF1A, CDH13, and RAR 32 ) on HR (adjusted for age), ER (adjusted for age and PR status), or PR (adjusted for age and ER status) status.

See http://www.biomedcentral.com/content/ supplementary/bcr1762-S3.doc

\section{Acknowledgements}

This work was supported by National Institutes of Health grants CA 80957 and CA 64602 and National Foundation for Cancer Research grant LF 2004-00009224HM.

\section{References}

1. Yang $X, Y a n L$, Davidson NE: DNA methylation in breast cancer. Endocr Relat Cancer 2001, 8:115-127.

2. Widschwendter $M$, Jones PA: DNA methylation and breast carcinogenesis. Oncogene 2002, 21:5462-5482.

3. Yuan J, Luo RZ, Fujii S, Wang L, Hu W, Andreeff M, Pan Y, Kadota $M$, Oshimura M, Issa J-P, et al.: Aberrant methylation and silencing of $A R H I$, an imprinted tumor suppressor gene whose function is lost in breast cancer. Cancer Res 2003, 63:4174-4180.

4. Fujii S, Luo RZ, Yuan J, Kadota M, Oshimura M, Dent SR, Kondo $Y$, Issa JP, Bast RC Jr, Yu Y: Reactivation of the silenced and imprinted alleles of ARHI is associated with increased histone $\mathrm{H} 3$ acetylation and decreased histone $\mathrm{H} 3$ lysine 9 methylation. Hum Mol Genet 2003, 12:1791-1800.

5. Dammann R, Yang G, Pfeifer GP: Hypermethylation of the CpG island of Ras association domain family $1 \mathrm{~A}$ (RASSF1A), a putative tumor suppressor gene from the 3p21.3 locus, occurs in a large percentage of human breast cancers. Cancer Res 2001, 61:3105-3109.

6. Krop IE, Sgroi D, Porter DA, Lunetta KL, LeVangie R, Seth P, Kaelin $\mathrm{CM}$, Rhei E, Bosenberg M, Schnitt S, et al.: HIN-1, a putative cytokine highly expressed in normal but not cancerous mammary epithelial cells. Proc Natl Acad Sci USA 2001, 98:9796-9801.

7. Fackler MJ, McVeigh M, Evron E, Garrett E, Mehrotra J, Polyak K, Sukumar S, Argani P: DNA methylation of RASSF1A, HIN-1, RAR-beta, Cyclin D2 and Twist in in situ and invasive lobular breast carcinoma. Int J Cancer 2003, 107:970-975.

8. Murata H, Khattar NH, Kang Y, Gu L, Li GM: Genetic and epigenetic modification of mismatch repair genes $\mathrm{hMSH} 2$ and hMLH1 in sporadic breast cancer with microsatellite instability. Oncogene 2002, 21:5696-5703.
9. Ferguson AT, Evron E, Umbricht CB, Pandita TK, Chan TA, Hermeking $\mathrm{H}$, Marks JR, Lambers AR, Futreal PA, Stampfer MR, Sukumar $\mathrm{S}$ : High frequency of hypermethylation at the 14-3-3 sigma locus leads to gene silencing in breast cancer. Proc Natl Acad Sci USA 2000, 97:6049-6054.

10. Du Y, Carling T, Fang W, Piao Z, Sheu JC, Huang S: Hypermethylation in human cancers of the RIZ1 tumor suppressor gene, a member of a histone/protein methyltransferase superfamily. Cancer Res 2001, 61:8094-8099.

11. Di Vinci A, Perdelli L, Banelli B, Salvi S, Casciano I, Gelvi I, Allemanni G, Margallo E, Gatteschi B, Romani M: p16(INK4a) promoter methylation and protein expression in breast fibroadenoma and carcinoma. Int J Cancer 2005, 114:414-421.

12. Hu XC, Loo WT, Chow LW: E-cadherin promoter methylation can regulate its expression in invasive ductal breast cancer tissue in Chinese woman. Life Sci 2002, 71:1397-1404.

13. Khan S, Kumagai T, Vora J, Bose N, Sehgal I, Koeffler PH, Bose S: PTEN promoter is methylated in a proportion of invasive breast cancers. Int J Cancer 2004, 112:407-410.

14. Wei M, Grushko TA, Dignam J, Hagos F, Nanda R, Sveen L, Xu J, Fackenthal J, Tretiakova M, Das S, Olopade OI: BRCA1 promoter methylation in sporadic breast cancer is associated with reduced BRCA1 copy number and chromosome 17 aneusomy. Cancer Res 2005, 65:10692-10699.

15. Mehrotra J, Vali M, McVeigh M, Kominsky SL, Fackler MJ, LahtiDomenici J, Polyak K, Sacchi N, Garrett-Mayer E, Argani P, Sukumar S: Very high frequency of hypermethylated genes in breast cancer metastasis to the bone, brain, and lung. Clin Cancer Res 2004, 10:3104-3109.

16. Widschwendter M, Siegmund KD, Muller HM, Fiegl H, Marth $C$, Muller-Holzner E, Jones PA, Laird PW: Association of breast cancer DNA methylation profiles with hormone receptor status and response to tamoxifen. Cancer Res 2004, 64:3807-3813.

17. Fiegl $H$, Millinger S, Goebel G, Muller-Holzner E, Marth C, Laird PW, Widschwendter M: Breast cancer DNA methylation profiles in cancer cells and tumor stroma: association with HER$2 /$ neu status in primary breast cancer. Cancer Res 2006, 66:29-33.

18. Colella S, Shen L, Baggerly KA, Issa JP, Krahe R: Sensitive and quantitative universal Pyrosequencing methylation analysis of CpG sites. Biotechniques 2003, 35:146-150.

19. Tost J, Dunker J, Gut IG: Analysis and quantification of multiple methylation variable positions in $\mathrm{CpG}$ islands by Pyrosequencing. Biotechniques 2003, 35:152-156.

20. Issa JP, Gharibyan V, Cortes J, Jelinek J, Morris G, Verstovsek S, Talpaz M, Garcia-Manero G, Kantarjian HM: Phase II study of low-dose decitabine in patients with chronic myelogenous leukemia resistant to imatinib mesylate. J Clin Oncol 2005, 23:3948-3956.

21. Yu YH, Xu FJ, Peng HQ, Fang XJ, Zhao SL, Li Y, Cuevas B, Kuo W-L, Gray JW, Siciliano M, et al:: NOEY2 (ARHI), an imprinted putative tumor suppressor gene in ovarian and breast carcinomas. Proc Natl Acad Sci USA 1999, 96:214-219.

22. Protocol used at National Cancer Institute $(\mathrm{NCl})$ and related information [http://cgap-mf.nih.gov/Protocols/Microdissection/ Overview.html]

23. Boumber YA, Kondo Y, Chen X, Shen L, Gharibyan V, Konishi K Estey E, Kantarjian H, Garcia-Manero G, Issa JP: RIL, a LIM gene on $5 q 31$, is silenced by methylation in cancer and sensitizes cancer cells to apoptosis. Cancer Res 2007, 67:1997-2005.

24. Estecio MR, Yan PS, Ibrahim AE, Tellez CS, Shen L, Huang TH, Issa JP: High-throughput methylation profiling by MCA coupled to CpG island microarray. Genome Res . 2007 Sep 4

25. Yang AS, Estecio MR, Doshi K, Kondo Y, Tajara EH, Issa JP: A simple method for estimating global DNA methylation using bisulfite PCR of repetitive DNA elements. Nucleic Acids Res 2004, 32:e38.

26. Campan M, Weisenberger DJ, Laird PW: DNA methylation profiles of female steroid hormone-driven human malignancies. Curr Top Microbiol Immunol 2006, 310:141-178.

27. Bardou VJ, Arpino G, Elledge RM, Osborne CK, Clark GM: Progesterone receptor status significantly improves outcome prediction over estrogen receptor status alone for adjuvant endocrine therapy in two large breast cancer databases. J Clin Oncol 2003, 21:1973-1979. 
28. Yan L, Yang X, Davidson NE: Role of DNA methylation and histone acetylation in steroid receptor expression in breast cancer. J Mammary Gland Biol Neoplasia 2001, 6:183-192.

29. Bryan BB, Schnitt SJ, Collins LC: Ductal carcinoma in situ with basal-like phenotype: a possible precursor to invasive basallike breast cancer. Mod Pathol 2006, 19:61 7-621.

30. Tischkowitz MD, Foulkes WD: The basal phenotype of BRCA1related breast cancer: past, present and future. Cell Cycle 2006, 5:963-967.

31. Krop I, Maguire P, Lahti-Domenici J, Lodeiro G, Richardson A Johannsdottir HK, Nevanlinna H, Borg A, Gelman R, Barkardottir $\mathrm{RB}$, et al:: Lack of HIN-1 methylation in BRCA1-linked and "BRCA1-like" breast tumors. Cancer Res 2003, 63:2024-2027.

32. Feki A, Irminger-Finger I: Mutational spectrum of p53 mutations in primary breast and ovarian tumors. Crit Rev Oncol Hematol 2004, 52:103-116.

33. Toyota M, Ohe-Toyota M, Ahuja N, Issa JP: Distinct genetic profiles in colorectal tumors with or without the CpG island methylator phenotype. Proc Natl Acad Sci USA 2000, 97:710-715.

34. Issa JP: Methylation and prognosis: of molecular clocks and hypermethylator phenotypes. Clin Cancer Res 2003, 9:2879-2881.

35. Rashid A, Issa JP: CpG island methylation in gastroenterologic neoplasia: a maturing field. Gastroenterology 2004, 127:1578-1588.

36. Brock MV, Gou MG, Akiyama Y, Muller A, Wu TT, Montgomery E, Deasel M, Germonpre P, Rubinson L, Heitmiller RF, et al.: Prognostic importance of promoter hypermethylation of multiple genes in esophageal adenocarcinoma. Clin Cancer Res 2003, 9:2912-2919.

37. Maruyama R, Toyooka S, Toyooka KO, Harada K, Virmani AK, Zochbauer-Muller S, Farinas AJ, Vakar-Lopez F, Minna JD, Sagalowsky A, et al:: Aberrant promoter methylation profile of bladder cancer and its relationship to clinicopathological features. Cancer Res 2001, 61:8659-8663.

38. Ogi K, Toyota M, Ohe-Toyota M, Tanaka N, Noguchi M, Sonoda T, Kohama G, Tokino T: Aberrant methylation of multiple genes and clinicopathological features in oral squamous cell carcinoma. Clin Cancer Res 2002, 8:3164-3171.

39. Wei SH, Chen CM, Strathdee G, Harnsomburana J, Shyu CR, Rahmatpanah F, Shi H, Ng SW, Yan PS, Nephew KP, et al:: Methylation microarray analysis of late-stage ovarian carcinomas distinguishes progression-free survival in patients and identifies candidate epigenetic markers. Clin Cancer Res 2002, 8:2246-2252.

40. Shen L, Toyota M, Kondo Y, Obata T, Daniel S, Pierce S, Imai K, Kantarjian HM, Issa JP, Garcia-Manero G: Aberrant DNA methylation of p57KIP2 identifies a cell cycle regulatory pathway with prognostic impact in adult acute lymphocytic leukemia. Blood 2003, 101:4131-4136. 